\title{
Inappropriate use of antibiotics in dentistry
}

Anirudha Agnihotry, BDS* Jassim Hassan Al-Langawi** Ankur Khanna, BDS***

*Preceptor, UCLA School of Dentistry, Los Angeles, California, USA

**Medical Student, College of Medicine and Medical Sciences, Arabian Gulf University, Manama, Bahrain

***Manipal College of Dental Sciences, Manipal University, India

Correspondence: anirudha.agnihotry@gmail.com

This is a summary of a Cochrane review assessing the effectiveness of interventions to relieve acute pain caused by irreversible pulpitis. It compares the use of antibiotics and analgesics against analgesics alone.

Fedorowicz Z, van Zuuren EJ, Farman AG, Agnihotry A, Al-Langawi JH. Antibiotic use for irreversible pulpitis. Cochrane Database of Systematic Reviews 2013, Issue 12. Art. No.: CD004969. DOI: 10.1002/14651858.CD004969. pub3.

Pulpitis is inflammation of the pulp tissue of the tooth. The precise symptoms for irreversible pulpitis vary but usually the patient presents with a history of spontaneous pain, aggravated by hot or cold stimulus, which lingers more than 30 seconds after stimulus is removed. ${ }^{1}$ Any tooth may be affected and it occurs as a sequela of dental caries, cracked tooth or trauma. The involved tooth is usually tender to percussion. The characteristic of irreversible pulpitis is a vital pulp which responds to cold and electric pulp testing. Treatment includes immediate complete removal of the pulpal tissue, i.e., pulpectomy; followed by cleaning and shaping of the root canal and obturating it with an inert material. ${ }^{2}$ Along with the pulpectomy, pain medication is required to deal with the procedural and post-procedural pain. ${ }^{3}$ Irreversible pulpitis, at least in the early phase, is not normally accompanied by the clinical signs of bacterial infection, i.e. swelling and tenderness of adjacent mucosa. This more generally manifests itself after the pulp has become necrotic and the infection spreads into the periapical region. Antibiotics are bactericidal or bacteriostatic or both, are used to control or eliminate bacterial infections, but the mode of action and extent to which antibiotics have an anti-inflammatory or analgesic effect in irreversible pulpitis remains unclear.

The systematic review being discussed was aimed at evaluating the effectiveness of pain medication alone against pain medication along with antibiotics to alleviate pain in irreversible pulpitis. This is the third update of the review. The first two searches were conducted in 2005 and 2009. The evidence on which this review is based was current as of 5 September 2013. One study involving 40 people with irreversible pulpitis (nerve damage) was included. There were two groups of 20 people; one group was treated with penicillin $500 \mathrm{mg}$, the other with placebo (no active ingredient) every six hours over a sevenday period. In addition, all of the participants received painkillers (ibuprofen and paracetamol (acetaminophen) combined with codeine). This study involved a small number of participants and the quality of the evidence for the different outcomes was rated as low. There is currently insufficient evidence to be able to decide if antibiotics help for this condition. This review highlights the need for more and better quality studies on the use of antibiotics for irreversible pulpitis. But, we can conclude through this study that antibiotics do not appear to significantly reduce toothache caused by irreversible pulpitis. Furthermore, there was no difference in the total number of ibuprofen or Tylenol tablets used over the study period between both groups. The administration of penicillin does not significantly reduce the pain perception, the percussion (tapping on the tooth) perception or the quantity of pain medication required by people with irreversible pulpitis. There was no reporting on adverse events or reactions. ${ }^{4}$

Still, the improper prescription of antibiotics in this respect is ubiquitous. A study was conducted of the members of the American Association of Endodontists (AAE) surveying their prescribing practices and found $16.7 \%$ of the specialist endodontists prescribed antibiotics for irreversible pulpitis. ${ }^{5}$ General dental practitioners are the first point of contact for patients and although one study conducted in Belgium reported a smaller proportion (4.3\%) of general dentists that continue to prescribe antibiotics for irreversible pulpitis ${ }^{6}$, a more recent survey conducted in Spain indicated that a substantial number $(86 \%)$ of respondents continue to do so. ${ }^{7}$ It is believed that the indiscriminate use of antibiotics may have added significantly to the increase in methicillinresistant Staphylococcus aureus (MRSA) infections with concomitant staggering cost implications. ${ }^{8}$ There is a steady increase in the number of MRSA infections and there is no estimate of how much unnecessary antibiotics are being prescribed in dentistry.

Building on this work, a new survey is being conducted to study the practices followed by clinicians to treat irreversible pulpitis. Please follow the link below, to access the survey.

https://docs.google.com/forms/d/1jKScibweUzPa6QbJCc bXMvKJRkS8cD9EngKnHrNuEqY/viewform

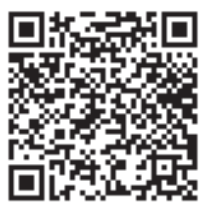




\section{REFERENCES}

1. Soames JV, Southam JC. Oral pathology. 3rd ed. Oxford: Oxford University Press; 1998. p. 51-70.

2. Walton RE, Torabinejad M. Endodontics: principles and practice. 4th ed. St Louis, Mo: Saunders Elsevier; 2009.

3. Pak JG, White SN. Pain prevalence and severity before, during, and after root canal treatment: a systematic review. J Endod. 2011 Apr;37(4):429-38.

4. Fedorowicz Z, Van Zuuren EJ, Farman AG, Agnihotry A, Al-Langawi JH. Antibiotic use for irreversible pulpitis. Cochrane Database Syst Rev. 2013 Dec 19;12:CD004969.

5. Yingling NM, Byrne BE, Hartwell GR. Antibiotic use by members of the American Association of Endodontists in the year 2000: report of a national survey. J Endod. 2002 May;28(5):396-404.

6. Mainjot A, D'hoore W, Vanheusden A, Van Nieuwenhuysen JP. Antibiotic prescribing in dental practice in Belgium. Int Endod J. 2009 Dec;42(12):1112-7.

7. Segura-Egea JJ, Velasco-Ortega E, Torres-Lagares D, Velasco-Ponferrada MC, Monsalve-Guil L, Llamas-Carreras JM. Pattern of antibiotic prescription in the management of endodontic infections amongst Spanish oral surgeons. Int Endod J. 2010 Apr;43(4):342-50.

8. Cox RA, Mallaghan C, Conquest C, King J. Epidemic methicillin-resistant Staphylococcus aureus: controlling the spread outside hospital. J Hosp Infect. 1995 Feb;29(2):10719. 